\title{
Fatores de Risco e Riscos Psicossociais no Trabalho: Definição e Implicações
}

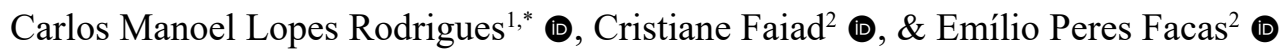 \\ ${ }^{1}$ Centro Universitário de Brasília, Brasília, DF, Brasil \\ ${ }^{2}$ Universidade de Brasília, Brasília, DF, Brasil
}

\begin{abstract}
RESUMO - O objetivo deste artigo teórico é analisar os conceitos de fatores de risco e riscos psicossociais no trabalho, bem como discuti-los a partir de duas perspectivas teóricas proeminentes no campo: o Modelo Demanda-Controle e a Psicodinâmica do Trabalho. Além das definições, são apresentadas as relações entre antecessores - fatores de risco, e consequentes - riscos psicossociais, e as implicações teóricas e práticas decorrentes da definição mais acurada dos termos. As conclusões apresentadas apontam para importância de delimitação conceitual entre fatores de risco e riscos psicossociais no trabalho como passo indispensável para o desenvolvimento de estratégias eficazes de avaliação e intervenção nos contextos de trabalho, principalmente pela adoção de uma postura preventiva com foco na identificação e controle dos fatores de risco prioritariamente.
\end{abstract}

PALAVRAS-CHAVE: riscos ocupacionais, saúde do trabalhador, saúde mental no trabalho

\section{Risk Factors and Psychosocial Risks at Work: Definition and Implications}

\begin{abstract}
The aim of this theoretical paper is to analyze the concepts of risk factors and psychosocial risks at work, as well as to discuss them from two prominent theoretical perspectives in the field: the Demand-Control Model and the Work Psychodynamics. In addition to the definitions, the relationships between predecessors - risk factors and consequent - psychosocial risks, and the theoretical and practical implications of the more precise definition of terms are presented. The conclusions presented point to the importance of conceptual delimitation between risk factors and psychosocial risks at work as an indispensable step for the development of effective strategies for evaluation and intervention in work contexts, mainly by adopting a preventive approach focused on the identification and control of risk factors.
\end{abstract}

KEYWORDS: occupational risks, worker's health, mental health at work

A partir das novas configurações que o trabalho vem assumindo, desde a década de 1970, os fatores que propiciam riscos psicossociais no trabalho passaram a representar tema emergente em diferentes contextos. Sua importância vem sendo reconhecida paulatinamente por governos, acadêmicos, organizações e sindicatos. Estas mudanças têm sido impulsionadas, por um lado, por novas formas de gestão com foco na otimização de recursos e flexibilidade e, por outro lado, pela constante inovação tecnológica, ambas situações emolduradas em um quadro de globalização intensa e constantes crises econômicas e sociais (Antunes, 2018;
Bouffartigue, 2012; Moreno-Jiménez, 2011). Em 1984, a Organização Internacional do Trabalho (OIT) publicou o primeiro documento oficial tratando especificamente dos riscos psicossociais no trabalho, alertando para sua incidência crescente e para necessidade de intervenção. Após essa publicação, foi construída uma agenda de pesquisa internacional, seguida de uma série de publicações da própria OIT e de outros organismos internacionais, no entanto no Brasil, tais investimentos mostram-se incipientes e com poucos avanços (Cardoso, 2014; Fischer, 2012; Rodrigues \& Faiad, 2019; Zanelli \& Kanan, 2018).

*E-mail: prof.carlos.manoel@gmail.com

- Submetido: 10/10/2019; Revisado: 04/11/2019; Aceito: 21/03/2020. 
Apesar de décadas de pesquisas produzidas na área, a definição de riscos psicossociais ainda encontra certa nebulosidade (Ruiz \& Araújo, 2012). Os termos riscos e fatores de risco são utilizados indistintamente (MorenoJiménez, 2011) e nem sempre corretamente compreendidos, ou até confundidos com outros termos como Qualidade de Vida no Trabalho e Clima Organizacional (Zanelli \& Kanan, 2018). Uma das consequências advindas dessa confusão conceitual é a dificuldade encontrada nos processos de avaliação e intervenção na área (Kortum et al., 2010, 2011; Weissbrodt \& Giauque, 2017).

A partir da análise destes conceitos, Guzmán (2011) destaca a necessidade de um esforço para definição mais precisa, que ao mesmo tempo incorpore a complexidade das configurações do trabalho contemporâneo e interliguem modelos teóricos diferentes. Em consonância com este pressuposto Neffa (2019a, 2019b), ao propor arcabouço teórico para análise dos riscos psicossociais no trabalho, elenca uma série de contribuições teóricas, de modelos de estresse a modelos de base sociológica que, apesar de abrangente, não se aprofunda na discussão da forma de interpretação dos fatores de risco e dos riscos psicossociais a partir destes modelos.

Em adição, a participação da psicologia neste campo ainda é tímida comparada com outras áreas. Moreno et al. (2015), ao analisarem a produção internacional entre 2000 e 2010 sobre riscos psicossociais no trabalho, relatam o predomínio de pesquisas conduzidas nos campos da medicina, epidemiologia e saúde pública. De forma similar, Rodrigues e Faiad (2019), a partir de uma análise bibliométrica da produção nacional sobre o tema, englobando os anos de 2008 e 2017, identificaram apenas oito estudos publicados em periódicos de psicologia, com maior concentração da produção nas áreas de enfermagem e saúde coletiva. Ambos estudos indicam a tendência de pesquisas descritivas e com a psicologia como pano de fundo das investigações.

Desta forma, este estudo teórico tem por objetivo analisar os conceitos de fatores de risco e de riscos psicossociais, bem como discuti-los a partir de dois modelos teóricos distintos e proeminentes na área: o Modelo Demanda-Controle (Karasek, 1979) e a Psicodinâmica do Trabalho (Dejours, 1992). Esta discussão busca identificar as bases de uso do modelo Demanda-Controle para os riscos psicossociais no trabalho, e, ainda, propor uma possibilidade de interpretação destes riscos a partir do arcabouço teórico da Psicodinâmica do Trabalho. Além disso, pretende-se contribuir no planejamento e na condução das ações de prevenção e controle dos fatores de risco psicossociais.

\section{RISCOS E FATORES DE RISCO PSICOSSOCIAIS NO TRABALHO}

Os termos risco e fator de risco, apesar de comumente utilizados no discurso acadêmico e profissional, nem sempre são aplicados na acepção conceitual originária, incorrendo, muitas vezes, em ambiguidades ou superposições. Um exemplo dessa confusão conceitual foi apresentado por Hansson (2005), que identificou cinco distintos significados de risco, a depender do contexto: (a) um evento não esperado ou um dano; (b) a causa de um evento não esperado; (c) a probabilidade de ocorrência de um evento não esperado; (d) a expectativa estatística de ocorrência de um evento; e (e) um fato sobre o qual se deve tomar uma decisão (Hansson, 2005). Ora o risco é identificado como um antecedente, ora como consequente.

Especificamente no campo dos riscos psicossociais, adota-se a concepção de risco como um dano à integridade física ou mental de um trabalhador (OIT, 1984), seja na forma de um transtorno ou doença, seja por lesão ou acidentes de trabalho. A concepção de risco se vincula, neste caso, ao adoecimento em si.

Já que os riscos psicossociais representam o dano produzido, os fatores que aumentam a probabilidade de ocorrência do dano são denominados fatores de risco psicossociais. Assim, fatores de risco são antecedentes dos riscos a eles associados, ressalva sendo feita que a relação de antecedência e consequência não é linearmente causal, pois os fatores de risco psicossociais interagem entre si, podendo assumir papel moderador, mediador, ou podem agir como proxy de riscos associados (Cortez et al., 2019; Kraemer et al., 2001).

Os fatores de risco psicossociais estão relacionados à interação dinâmica entre os indivíduos e seu trabalho, compreendendo o desempenho profissional; o controle e autonomia - inclusive em relação às funções, tarefas e atividades realizadas; a forma de organização dos esquemas de produção; a jornada e intensidade do trabalho; às características organizacionais e o ambiente interno e externo no qual as organizações de trabalho se inserem (Ahumada \& Martínez, 2011; Portuné, 2012). Estes fatores, quando negativos, relacionam-se e antecedem a um grande número de agravos a saúde do trabalhador, agravos estes que incluem transtornos mentais, acidentes de trabalho, suicídio e abuso de substância, por exemplo (Benavides et al., 2002; Fischer, 2012; Harvey et al., 2017; Stansfeld \& Candy, 2006).

Os riscos psicossociais, nesta concepção, englobam, portanto, a produção de efeitos deletérios sobre a saúde dos trabalhadores, resultantes da exposição aguda ou crônica aos fatores de risco psicossociais (Moreno-Jiménez, 2011). Em uma pesquisa com especialistas de 21 países dos cinco continentes, Kortum et al.(2010) identificaram os principais agravos a saúde dos trabalhadores caracterizáveis como riscos psicossociais (Tabela 1). 
Tabela 1

Riscos psicossociais mais comuns

\begin{tabular}{ll}
\hline Âmbito & Agravos à saúde \\
\hline Saúde física & Cardiocirculatórios \\
& Gastrointestinais \\
& Alterações Musculoesqueléticas \\
& Dores de cabeça / enxaquecas \\
& Sintomas respiratórios e dermatológicos \\
& Acidentes e lesões \\
& Diabetes \\
& Úlceras \\
Cânceres específicos \\
Daúde mental & Depressão, ansiedade, problemas emocionais \\
Suicídio / comportamentos suicidas \\
Transtornos mentais em geral \\
Abuso de substância \\
Tabagismo \\
Obesidade \\
\hline
\end{tabular}

Nota: Adaptado de Kortum et al. (2010, p. 234).

A revisão conduzida por Zanelli e Kanan (2018) aponta entre riscos e fatores de riscos mais comuns - o assédio moral, sexual e a violência no trabalho; a adição ao trabalho; o consumo abusivo de substâncias; a corrupção; o estresse; o burnout; os transtornos do humor e de ansiedade; e o suicídio no trabalho, sendo a severidade dos danos ou agravos definidos pela intensidade e tempo de exposição aos fatores de risco. Em contrapartida, quando da existência de fatores psicossociais positivos, ou protetivos, as consequências, segundo os autores, incluem o bem-estar no trabalho; a satisfação e engajamento; práticas positivas de liderança, por exemplo. Apesar do detalhamento apresentado pelos autores, a indicação do assédio moral e/ou sexual e da violência no trabalho como riscos e não como fatores de risco pode ser problematizada, uma vez que ora a exposição a estas situações pode ser considerada como um fator de risco, ora estes comportamentos podem ser analisados como produtos de um contexto de trabalho adoecido.

Em busca de uma melhor delimitação dos fatores de risco psicossociais, Ahumada e Martínez (2011) propõem uma classificação quanto à natureza destes fatores. Esta classificação apresenta sete áreas: (1) desempenho profissional; (2) direção; (3) organização e função; (4) tarefas e atividades; (5) meio ambiente de trabalho; (6) jornada de trabalho; e (7) organização e contexto social. Tais áreas não constituem fatores de risco por si só, mas dependem do processo de interiorização da vivência destes fatores no ambiente laboral (Uribe, 2015). Assim, cada trabalhador, a depender de suas características individuais, como por exemplo idade, gênero e fatores de personalidade (Gartzia et al., 2018; Havermans et al., 2018; Ilić et al., 2017), percebe e vivencia a exposição aos fatores de risco de forma mais ou menos intensa.
Isto posto, pode-se recuperar o conceito de fatores de riscos psicossociais no trabalho proposto originalmente pela OIT (1984) como a interação entre as características do trabalho e da organização e as características dos trabalhadores, cuja percepção por parte destes últimos podem repercutir na saúde e no rendimento e satisfação com trabalho. Este conceito enfatiza a interação entre níveis diferentes de análise (Kortum \& Leka, 2014), característica inerente a fenômenos organizacionais complexos que implica na necessidade de reconhecimento das relações dinâmicas que se constituem nas organizações sob a pena de realização de estudos e intervenções superficiais e ineficazes (Puente-Palacios et al., 2016).

A característica perceptual envolvida na exposição aos fatores de risco psicossociais em adição aos diferentes níveis que abarcam o fenômeno implica na necessidade de modelos teóricos que incluam estas dimensões para compreensão de como os fatores organizacionais e do trabalho interagem com os fatores individuais gerando essa percepção. Dentre os modelos aplicáveis a este contexto, o Modelo DemandaControle e a psicodinâmica do trabalho atendem a esta necessidade. O Modelo Demanda-Controle apresenta larga utilização neste campo de pesquisa (Dollard et al., 2007; Lorente \& Yeves, 2016; Rodrigues \& Faiad, 2019; Zoni \& Luchini, 2012), inclusive em estudos experimentais (O’Donnell et al., 2015), além de basear outros modelos. A Psicodinâmica do Trabalho constitui-se como uma abordagem relevante nos estudos da saúde do trabalhador, além de possibilitar a discussão quanto a experiência subjetiva dos trabalhadores (Dashtipour \& Vidaillet, 2017; Duarte \& Mendes, 2013; Machado \& Macêdo, 2016) a partir da exposição aos fatores de riscos e aos mecanismos subjacentes a produção dos riscos psicossociais no trabalho (Jacinto \& Tolfo, 2017). 


\section{MODELO DEMANDA-CONTROLE}

Desenvolvido por Karasek (1979), o Modelo DemandaControle foi proposto inicialmente para o estudo do estresse laboral e define duas dimensões identificáveis no trabalho. Por um lado, existe uma demanda, entendida como as exigências físicas, cognitivas e emocionais de um determinado contexto e, por outro, o controle, representado pelo grau de decisão que os sujeitos podem exercer na situação (Araújo et al., 2003).

O modelo prevê que o nível de estresse experienciado em determinada atividade laboral seja definido pela relação entre a demanda da atividade e controle que o indivíduo possui sobre seu trabalho. Karasek (1979) indica que a relação entre as dimensões implica em quatro possibilidades: trabalhos com baixa exigência (baixa demanda e alto controle); trabalho ativo (alta demanda e alto controle); trabalho passivo (baixa demanda e baixo controle); e trabalhos com alta exigência (alta demanda e baixo controle). No modelo, o controle exerce função moderadora da experiência de estresse, uma vez que nas atividades de alta demanda com alto controle o estresse é vivenciado de forma mais salutar que em situações de alta demanda e baixo controle.

Tanto situações com alto controle quanto com alta demanda tendem a serem percebidas de forma mais intensa pelos trabalhadores. A interação entre estas condições, conforme estudos realizados em diversos ambientes laborais, é identificada como determinante, seja na experiência de estresse (Luchman \& González-Morales, 2013), seja nas reações fisiológicas, como ritmo cardíaco e produção de alfa-amilase salivar (O’Donnell et al., 2015).

Assim, o estresse é concebido como a experiência das situações onde as demandas suplantam o controle, e não como uma categoria nosológica independente, como no caso dos transtornos de estresse agudo, que é o resultado da vivência de situações extremas. O estresse, nesta perspectiva, é avaliado pela mensuração das demandas e do controle em cada contexto de trabalho (Bell et al., 2017), ou seja, dos fatores geradores da experiência, diferindo substancialmente de outras propostas baseadas em alterações comportamentais ou fisiológicas, que se configuram como medidas das consequências da exposição dos trabalhadores às situações estressoras, mas sem a identificação dos fatores geradores (Ganster \& Rosen, 2013).
A partir do modelo Demanda-Controle foram desenvolvidos instrumentos de avaliação com foco nas dimensões demanda e controle, que vão desde o bastante conhecido Job Content Questionaire (JCQ; Karasek, 1985; Karasek et al., 1998), até versões baseadas no JCQ incluindo dimensões que buscam alcançar a diversidade de situações laborais que compõem as configurações contemporâneas do trabalho, abarcando o conflito trabalho-família, a exposição à violência e fatores relativos ao entorno social das organizações. Nesta linha, o Working Conditions and Control Questionnaire (WOCCQ; Hansez \& Keyser, 1999) e a Escala de Avaliação de Estressores Psicossociais no Contexto Laboral (Ferreira et al., 2015), por exemplo, foram desenvolvidos a partir do Modelo Demanda-Controle e do JCQ em uma perspectiva de avaliação de fatores de riscos psicossociais no trabalho.

Por suas características facilitarem a operacionalização nos contextos de trabalho, o modelo Demanda-Controle tem sido o mais utilizado na análise dos fatores de risco psicossociais no trabalho (Rodrigues \& Faiad, 2019; Zoni \& Luchini, 2012). Para tanto, considera-se que os descompassos entre as demandas laborais e externas e o controle que o trabalhador pode ou é capaz de exercer configuram-se como fatores de risco psicossociais. Esta proposta permite incluir as demandas emergentes como automação constante, instabilidade no emprego, percepção de futuro e exposição à violência (Kortum et al., 2011; OIT, 2010). Estas características tornam o modelo DemandaControle, do ponto de vista metodológico, mais próximo a estudos de caráter epidemiológico, correlacionais e longitudinais (Dong et al., 2018; Lesener et al., 2019; Rodrigues \& Faiad, 2019).

A despeito de sua difusão, o modelo Demanda-Controle apresenta algumas limitações, como salientado por Lorente e Yeves (2016), na aplicabilidade a contextos laborais onde as demandas sejam muito específicas e por desconsiderar variáveis que podem atuar na experiência de trabalho, por exemplo, liderança ou mesmo exigências emocionais. Outra limitação diz respeito aos instrumentos de medida desenvolvidos a partir do modelo, que apresentam características psicométricas mais favoráveis para avaliação das demandas que do controle (Bell et al., 2017).

\section{PSICODINÂMICA DOTRABALHO}

A Psicodinâmica do Trabalho surgiu na década de 1980, proposta pelo médico e psicanalista francês Christophe Dejours. A proposta inicial da abordagem foi investigar quais estratégias de mediação os trabalhadores utilizavam no confronto com o real do trabalho e como elas se relacionavam ao prazer ou sofrimento no trabalho (Brant \& Minayo-Gomez, 2004; Duarte \& Mendes, 2013).
Com difusão relevante no contexto acadêmico nacional (Machado \& Macêdo, 2016; Merlo \& Mendes, 2009) este modelo permite uma leitura de fatores de risco e de riscos psicossociais no trabalho por meio de seus conceitos chave.

O início do desenvolvimento da Psicodinâmica do Trabalho, ainda na década de 1980, se dá sob a influência da Psicopatologia do Trabalho, em especial os estudos 
conduzidos por Le Guillant (Dejours, 1992; Souza \& Athayde, 2006). Neste primeiro momento, Dejours (1992) procurava compreender o sofrimento e o modo como os trabalhadores lidavam com este. A partir dos anos 1990, a abordagem passa a se desenvolver a partir da aproximação com a Psicanálise, a Ergonomia da Atividade e a Teoria Crítica da Escola de Frankfurt, com especial interesse nas discussões sobre os processos de subjetivação dos trabalhadores com relação às vivências de prazer-sofrimento decorrentes do trabalho, novas organizações do trabalho, o sentido das vivências e as estratégias de mediação decorrentes destas. Trata-se, então, de uma abordagem interdisciplinar constituída a partir do diálogo entre essas diferentes vertentes.

Um dos focos de estudo da Psicodinâmica do Trabalho é a investigação dos mecanismos psíquicos mobilizados nas situações de trabalho, recorrendo assim a conceitos originários da psicanálise, como o conceito de mecanismo de defesa. A psicodinâmica do trabalho traz como uma de suas premissas que o trabalho é um lugar de investimento pulsional. Assim, pode ser fonte de prazer, quando o trabalhador se realiza, vê sentido no que faz e é reconhecido, quando o trabalho é livre e criativo, mas pode, também, ser fonte de sofrimento, quando é infrutífero e desgastante, quando não há reconhecimento e liberdade (Alderson, 2004; Duarte \& Mendes, 2013).

Este sofrimento pode levar o trabalhador a utilizar mecanismos de defesa individuais e/ou coletivos ou a mobilizar-se, individual ou coletivamente. Quando a mobilização permite a emancipação e potencializa a saúde dos trabalhadores, tem-se o sofrimento criativo (Dejours, 1992, 2012). Em oposição, quando não há possibilidades, ou elas são reduzidas, para mobilização de estratégias coletivas ou individuais de enfrentamento do sofrimento, há a vivência do sofrimento patogênico (Facas, 2013; Martins, 2008). O sofrimento patológico também se estabelece quando as estratégias mobilizadas alienam ou desconectam o trabalhador do sofrimento, potencializando o adoecimento (Dejours, 2012).

O sofrimento patológico é uma forma de experiência mediata da exposição aos fatores de riscos psicossociais que antecedem o adoecimento no trabalho. Considerando isto, Facas (2013) desenvolve o Protocolo de Avaliação dos Riscos Psicossociais no Trabalho (PROART), a partir das contribuições teóricas da Psicodinâmica do Trabalho. A compreensão dos fatores de risco e riscos psicossociais é possível ao se considerar fatores de risco com resultantes da interação entre configurações e condições do trabalho, as estratégias mobilizadas ou não, e o sofrimento patológico vivenciado (Figura 1). Já os riscos psicossociais são o desfecho da exposição ao sofrimento patogênico, caracterizado pelo adoecimento físico e mental.

Como apontado por Facas (2013), uma das dificuldades para avaliação de fatores de riscos e de riscos psicossociais, nesta orientação, é a articulação entre os diversos construtos que compõem o modelo. Além disso, o próprio uso dos termos fatores de risco e riscos psicossociais é questionado no campo da psicodinâmica do trabalho, principalmente em função da possibilidade do foco exclusivo nos 'fatores humanos" e permitir a culpabilização dos trabalhadores pelo processo de adoecimento (Dejours, 1995; Gollac \& Bodier, 2011) e a uma menor tendência ao uso de instrumentos psicométricos (Jacinto \& Tolfo, 2017) estratégia comum nos estudos nos estudos sobres riscos psicossociais no trabalho.

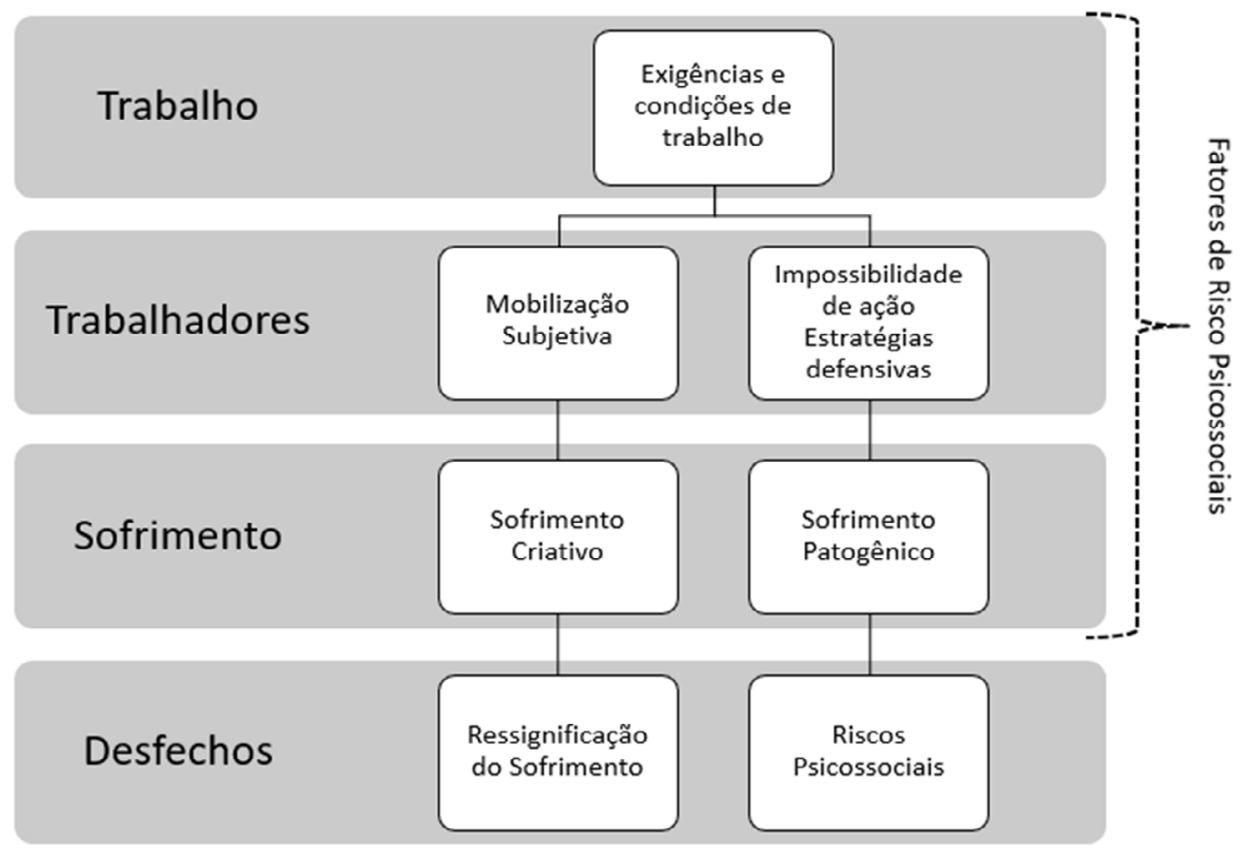

Figura 1. Proposição de Concepção de Fatores de Risco e Riscos Psicossociais no Trabalho a partir da Psicodinâmica do Trabalho 
Tal questionamento sobre a utilização dos termos fatores de riscos e riscos psicossociais e a decorrente preocupação com a possibilidade de um deslocamento de responsabilidade para os próprios sujeitos pelos processos de adoecimento são pertinentes e merecem olhares e discussões cuidadosas sobre o tema. Contudo, esse cuidado não pode servir de obstáculo para trazer ao debate os estudos de Christophe Dejours sobre trabalho, saúde e processos (inter)subjetivos e seu potencial de contribuição para a concepção de riscos e fatores de riscos no trabalho. Não se trata de adequar forçosamente os conceitos fundamentais da abordagem, criando uma falsa impressão de que a dinâmica entre trabalho e processo de adoecimento pode ser explicada apenas por elementos objetivos presentes nas diferentes organizações do trabalho e, deste modo, que o risco poderia ser eliminado a partir de uma intervenção direta de um suposto especialista. Trata-se justamente de reconhecer que os avanços teóricos podem fornecer subsídios para uma nova visão sobre riscos psicossociais, como discutido em Facas (2018).

As novas formas de organização do trabalho e os novos modelos de gestão/management - com características como o culto à qualidade total e as avaliações individualizadas de desempenho - e seus efeitos deletérios na saúde dos trabalhadores têm sido discutidos largamente em pesquisas nacionais - ver Merlo e Mendes (2009) - e internacionais, bem como são temas recorrentes na produção teórica da Psicodinâmica do Trabalho (Dejours, 2008; 2015). Esses modelos de organização e gestão do trabalho têm trazido como consequência a desarticulação dos coletivos de trabalho e a desconfiança entre os trabalhadores decorrente da lógica de competição/concorrência entre os sujeitos, minando as dinâmicas de reconhecimento tal como preconizado pela teoria. Como apresentado em Facas (2018),

a avaliação das relações saúde-trabalho demanda pensar as [...] as implicações da relação organização do trabalho, gestão e trabalhador - articulando a organização do trabalho e os processos de subjetivação manifestos nas vivências de prazer-sofrimento, nas estratégias de ação para mediar contradições, nas patologias sociais e na saúde. Insistir nas análises individuais e coletivas das dicotômicas relações prazer-sofrimento, entendendo o trabalho como mediador insubstituível da realização pessoal no campo social. (p. 50)

Ao entendermos que para a Psicodinâmica do Trabalho o sofrimento é inerente ao trabalho e que é justamente o destino que este sofrimento terá - criativo ou patogênico - que será determinante na dinâmica saúde-adoecimento, podemos afirmar que todo trabalho implica em risco. Deste modo, a lógica da discussão de fatores de riscos e riscos psicossociais não deve ser centrada na possibilidade da eliminação do risco - o que, claramente, seria impossível. Entende-se que a saúde não pressupõe a ausência de sofrimento no trabalho, mas as possibilidades de o trabalhador transformá-lo. Para tal, é necessário que o trabalhador seja reconhecido em seu esforço e investimento na tarefa, possibilitando assim que o sofrimento vivenciado tenha sentido.

\section{IMPLICAÇÕES}

Considerando a distinção e a relação entre riscos e fatores de risco, algumas consequências podem ser identificadas, sendo a primeira relativa aos processos de avaliação baseados nesses construtos. Avaliar fatores de risco e avaliar riscos envolve focos diferentes e estratégias diferentes, com consequências e propósitos diversos.

A avaliação de fatores de risco, na concepção aqui delimitada, pressupõe a avaliação das condições antecessoras dos riscos, ou seja, na avaliação das configurações do trabalho em seu contexto micro e macro, que propiciam os agravos à saúde do trabalhador. Esta avaliação, para ser completa, deverá abarcar múltiplos níveis e incluir variáveis mediadoras e moderadoras, constituindo-se aqui um desafio metodológico e prático (Kortum \& Leka, 2014).

Esta afirmação apresenta uma dificuldade, que não é específica da avaliação de fatores de risco, mas que está presente nas áreas onde os fenômenos por sua natureza emergem na interação entre diferentes níveis organizacionais (Puente-Palacios et al., 2016), uma vez que a realização de avaliações que contemplem os níveis micro e macro são poucas vezes levadas em consideração. Avaliar fatores de risco demanda, portanto, o desenvolvimento de instrumental que permita acessar os níveis do indivíduo, da organização e do contexto social onde a organização se insere (Cardoso, 2014), e em uma abordagem ideal, no contexto micro e macrossocial (Kortum \& Leka, 2014).

Quando da mudança do foco da avaliação dos fatores de riscos para a avaliação dos riscos, sem que a primeira seja mapeada, pode-se incorrer no erro de não se identificar determinado agravo à saúde como relacionado com fatores de risco psicossociais, uma vez que nem todo risco psicossocial é caracterizável como um transtorno mental (Stansfeld \& Candy, 2006; Kortum et al., 2010). Tal posição, possibilita, ainda, que nos concentremos apenas em determinados riscos, como depressão ou burnout por serem mais facilmente reconhecíveis em sua relação com fatores de risco psicossociais, mascarando a influência destes fatores nos acidentes, por exemplo (Moreno-Jiménez, 2011). A postura preventiva na pesquisa e na intervenção neste campo será orientada para avaliação dos fatores de risco prioritariamente (Cardoso, 2014; Fischer, 2012), levando em consideração os níveis que compõem o fenômeno, evitando avaliações com foco exclusivo no indivíduo como fator problema, ou da organização descontextualizada de seu ambiente. 
Apesar do Modelo Demanda-Controle embasar grande parte da produção da pesquisa nesta área (Lorente \& Yeves, 2016; Rodrigues \& Faiad, 2019; Zoni \& Luchini, 2012), a escolha de um modelo teórico deve levar em consideração as potencialidades e limitações de cada um. Se, por um lado, o modelo Demanda-Controle apresenta maior suporte empírico e apresenta construtos com maior possibilidade de operacionalização para o desenvolvimento de medidas, por outro a Psicodinâmica do Trabalho oferece ferramentas de análise com maior propensão a avaliar as particularidades de cada contexto laboral e seus efeitos nos trabalhadores.

Entretanto, como salientado por Neffa (2019a, 2019b), ante a complexidade do tema, a integração de perspectivas teóricas diferentes se apresenta como necessária. Este movimento de integração pode ser observado no trabalho de Facas $(2013,2018)$ a partir do desenvolvimento do PROART, que parte do referencial teórico da Psicodinâmica do Trabalho para operacionalizar a avaliação de fatores de risco psicossociais.

$\mathrm{O}$ instrumento congrega dimensões relativas à organização e gestão do trabalho, com dimensões focadas no sofrimento patogênico no trabalho e danos físicos e psicossociais, com exemplos de utilização de profissionais de atendimento pré-hospitalar (Pacheco \& Silva, 2018) a profissionais da área de educação (Araujo \& Oliveira, 2019). Esta aproximação entre modelos teóricos pode auxiliar na superação dos principais gargalos na gestão dos riscos psicossociais no trabalho: a correta identificação dos fatores de risco e o desenvolvimento de ações e políticas preventivas eficientes (Kortum \& Leka, 2014; Kortum et al., 2010).

A pesquisa no campo, portanto, necessita avançar na compreensão da forma que a exposição aos fatores de risco psicossociais produz os agravos à saúde do trabalhador em termos dos mecanismos psicológicos subjacentes ao adoecimento, a partir da natureza perceptual destes fatores. Além da integração de modelos teóricos, também é necessário o desenvolvimento de estratégias metodológicas que integrem múltiplas fontes de informação e indicadores, além do investimento em estudos longitudinais (Neffa, 2019a; Rodrigues \& Faiad, 2019).

\section{CONSIDERAÇÕES FINAIS}

O reconhecimento dos riscos psicossociais representa um avanço na proteção à saúde o trabalhador (Dollard et al., 2007; Ruiz \& Araújo, 2012). Entretanto o desconhecimento ainda presente sobre estes riscos é uma barreira para esta efetiva proteção (Kortum et al., 2010; OIT, 2010). Some-se a utilização dos conceitos de risco e fatores de risco como equivalentes, aumenta-se a chance de ações que apenas tangenciem os problemas decorrentes da organização contemporânea do trabalho (Moreno-Jiménez, 2011).

Delimitar conceitualmente fatores de risco e riscos psicossociais no trabalho tem implicações teóricas e empíricas relevantes, principalmente para o desenvolvimento de estratégias eficazes de avaliação e intervenção nos contextos de trabalho. A efetiva promoção do trabalho como fonte de emancipação e de dignidade humana passa, necessariamente, pela identificação e controle dos fatores de risco (Zanelli \& Kanan, 2018).

É necessário, entretanto, ressaltar que este artigo apresenta limitações relativas a escolha dos modelos teóricos apresentados, onde se procurou evidenciar a possibilidade de aplicação de dois modelos, mais pela sua representatividade que por uma exposição exaustiva de todas as concepções teóricas do campo. O modelo Demanda-Controle é apresentado em sua forma básica de aplicação no contexto deste ensaio por sua representatividade e evidências que lhe alicerçam; já a Psicodinâmica do Trabalho, por sua difusão no contexto brasileiro. Todavia, ambos possuem limitações e interfaces com outras proposições teóricas que merecem atenção, mas que extrapolam os objetivos ora delineados.

Por fim, esta apresentação conceitual pretende auxiliar na atenuação da polissemia que estes termos têm assumido. Indica-se, ainda, como agenda de pesquisa, o aprofundamento quanto à forma pela qual os diversos tipos de fatores de risco interagem entre si, e quais mecanismos atuam na produção dos riscos psicossociais a partir da exposição aos fatores de risco.

\section{REFERÊNCIAS}

Ahumada, H. T., \& Martínez, M. R. (2011) Conceptos básicos em la evaluacíon del riesgo psicossocial em los centros de trabajo. In A. J. García \& A. C. Ávila, (Eds.), Reflexiones teóricoconceptulaes de lo psicossocial em el trabajo (pp. 95-112). Juan Pablos Editor.

Alderson, M. (2004). La psychodynamique du travail: Objet, considérations épistémologiques, concepts et prémisses théoriques. Santé Mentale au Québec, 29(1), 243-260. https:// doi.org/10.7202/008833ar
Antunes, R. (2018). O privilégio da servidão: O novo proletariado de serviço na era digital. Boitempo Editorial.

Araújo, L. K. R., \& Oliveira, S. S. (2019). Mapeamento dos riscos psicossociais no SAMU/DF. Psicologia: Ciência e Profissão, 39, e184126. https://doi.org/10.1590/1982-3703003184126

Araújo, T. M, Graça, C. C., \& Araújo, E. (2003). Estresse ocupacional e saúde: Contribuições do Modelo DemandaControle. Ciência \& Saúde Coletiva, 8(4), 991-1003. https:// doi.org/10.1590/S1413-81232003000400021 
Bell, C., Johnston, D., Allan, J., Pollard, B., \& Johnston, M. (2017). What do Demand-Control and Effort-Reward work stress questionnaires really measure? A discriminant content validity study of relevance and representativeness of measures. British Journal of Health Psychology, 22(2), 295-329. https://doi. org/10.1111/bjhp.12232

Benavides, F. G, Benach, J., \& Muntaner, C. (2002). Psychosocial risk factors at the workplace: is there enough evidence to establish reference values?. Journal of Epidemiology and Community Health, 56(4) 244-245. https://doi.org/10.1136/ jech.56.4.244

Bouffartigue, P. (2012). Les risques psychosociaux. Quels enjeux de démocratie et de justice au travail? [Conference presentation]. Second forum de l'Association Internationale de Sociologie, Buenos Aires. https://halshs.archives-ouvertes. $\mathrm{fr} /$ halshs-00726017

Brant, L. C., \& Minayo-Gomez, C. (2004). A transformação do sofrimento em adoecimento: do nascimento da clínica à psicodinâmica do trabalho. Ciência \& Saúde Coletiva, 9(1), 213-223. https://doi.org/10.1590/S1413-81232004000100021

Cardoso, A. C. M. (2014). Indicadores sobre riscos psicossociais no trabalho. In M. A. Silveira (Ed.), Aspectos psicossociais e sustentabilidade em organizações: Saúde, segurança e qualidade de vida no trabalho (pp. 130-144). CTI Renato Archer.

Cortez, P. A., Zerbini, T., \& Veiga, H. M. S. (2019). Work context and burnout: Confirmation of moderators from meta-analysis evidence. Revista Psicologia Organizações e Trabalho, 19(4), 755-761. https://doi.org/10.17652/rpot/2019.4.17499

Dashtipour, P., \& Vidaillet, B. (2017). Work as affective experience: The contribution of Christophe Dejours' "psychodynamics of work'. Organization, 24(1), 18-35. https://doi. org/10.1177/1350508416668191

Dejours, C. (1992). A loucura do trabalho: Estudos de psicopatologia do trabalho $\left(5^{\mathrm{a}}\right.$ ed.). Cortez-Oboré. (Original published in 1980)

Dejours, C. (1995). Le facteur humain. Paris: PUF.

Dejours, C. (2008). A avaliação do trabalho submetida a prova do real - Críticas aos fundamentos da avaliação. In L. Sznelwar, \& F. Mascia (Ed.), Trabalho, tecnologia e organização (pp. 31-90). Blucher.

Dejours, C. (2012). Trabalho vivo: Trabalho e emancipação. Paralelo 15.

Dejours, C. (2015). Organização do trabalho e saúde mental: Quais são as responsabilidades do manager? In K. B. Macêdo (Ed.), $O$ diálogo que transforma: A clínica psicodinâmica do trabalho (pp. 223-233). PUC Goiás.

Dollard, M., Skinner, N., Tuckey, M. R., \& Bailey, T. (2007) National surveillance of psychosocial risk factors in the workplace: An international overview. Work \& Stress, 21(1), 1-29. https://doi.org/10.1080/02678370701254082

Dong, L., Eaton, W. W., Spira, A. P., Agnew, J., Surkan, P. J., \& Mojtabai, R. (2018). Job strain and cognitive change: The Baltimore Epidemiologic Catchment Area follow-up study. Occupational and Environmental Medicine, 75(12), 856-862. https://doi.org/10.1136/oemed-2018-105213

Duarte, F. S., \& Mendes, A. M. (2013). Notas sobre o percurso teórico da psicodinâmica do trabalho. In L. G. Freitas (Ed.), Prazer e sofrimento no trabalho docente: Pesquisas brasileiras (pp. 13-24). Juruá.

Facas, E. P. (2013). Protocolo de Avaliação dos Riscos Psicossociais no Trabalho: Contribuições da Psicodinâmica do Trabalho [Tese de doutorado]. Repositório Institucional da UnB. https:// repositorio.unb.br/handle/10482/15420

Facas, E. P. (2018). Avaliação de riscos psicossociais no trabalho: Contribuições da Psicodinâmica do Trabalho. In M. L. G. Schmidt, M. F. Castro, \& M. M. Casadore (Eds.), Fatores psicossociais e o processo saúde/doença no trabalho - Aspectos teóricos, metodológicos, interventivos e preventivos (pp. 4153). FiloCzar.

Ferreira, M. C., Milfont, T. L., Corrêa, A. P., Fernandes, H. A., Almeida, S. P., \& Mendonça, H. (2015). Escala para Avaliação de Estressores Psicossociais no Contexto Laboral: Construção e evidências de validade. Psicologia: Reflexão e Crítica, 28(2), 340-349. https://doi.org/10.1590/1678-7153.201528214

Fischer, F. M. (2012). Relevance of psychosocial factors at work for workers' health. Revista de Saúde Pública, 46(3) 401-406. https://doi.org/10.1590/S0034-89102012000300001

Ganster, D. C., \& Rosen, C. C. (2013). Work stress and employee health: A multidisciplinary review. Journal of Management, 39, 1085-1122. https://doi.org/10.1177/0149206313475815

Gartzia, L., Pizarro, J., \& Baniandres, J. (2018). Emotional androgyny: A preventive factor of psychosocial risks at work? Frontiers in Psychology, 9, 2144. https://doi.org/10.3389/ fpsyg.2018.02144

Gollac, M., \& Bodier, M. (2011). Collège d'expertise sur le suivi des risques psychosociaux au travail. Mesurer les facteurs psychosociaux de risque au travail pour les maîtriser. [Rapport du Collège d'expertise sur le suivi des risques psychosociaux au travail, faisant suite à la demande du ministre du Travail, de l'Emploi et de la Santé, 42]. https://www.vie-publique.fr/ sites/default/files/rapport/pdf/114000201.pdf

Guzmán, L. G. D. (2011). Factores psicosociales: Uma crítica a su definición. In A. J. García \& A. C. Ávila (Eds.), Reflexiones teórico-conceptulaes de lo psicossocial em el trabajo (pp. 77-93). Juan Pablos Editor.

Hansez, I., \& Keyser, V. (1999). El WOCCQ: Una nueva herramienta en el estuche de instrumentos contra los estresores laborales. El caso de los trabajadores de servicios públicos en Bélgica. Revista de Psicología del Trabajo y de las Organizaciones, 15(2), 173-198. https://journals.copmadrid. org/jwop/files/51263.pdf

Hansson, S. O. (2005). Seven myths of risk. Risk Management: An International Journal, 7(2), 7-17. https://www.jstor.org/ stable/3867684

Harvey, S. B., Modini, M., Joyce, S., Milligan-Saville, J. S., Tan, L., Mykletun, A., Bryant, R. A., Christensen, H., \& Mitchell, P. B. (2017). Can work make you mentally ill? A systematic meta-review of work-related risk factors for common mental health problems. Occupational Environmental Medicine, 74(4), 301-310. https://doi.org/10.1136/oemed-2016-104015

Havermans, B. M., Boot, C. R., Hoekstra, T., Houtman, I. L., Brouwers, E. P., Anema, J. R., \& van der Beek, A. J. (2018). The association between exposure to psychosocial work factors and mental health in older employees, a 3-year follow-up study. International Archives of Occupational and Environmental Health, 91(1), 57-66. https://doi.org/10.1136/ oemed-2016-104015

Ilić, I. M., Arandjelović, M. Ž., Jovanović, J. M., \& Nešić, M. M. (2017). Relationships of work-related psychosocial risks, stress, individual factors and burnout-Questionnaire survey among emergency physicians and nurses. Medycyna Pracy, 68(2), 167-178. https://doi.org/10.13075/mp.5893.00516

Jacinto, A., \& Tolfo, S. R. (2017). Riscos psicossociais no trabalho: Conceitos, variáveis e instrumentos de pesquisa. Perspectivas em Psicologia, 21(1), 35-59. https://doi.org/10.14393/ PPv21n1a2017-04

Karasek, R. (1985). Job Content Instrument: Questionnaire and User's Guide, revision 1.1. University of Southern California.

Karasek, R. A. (1979). Job demands, job decision latitude, and mental strain: Implications for job redesign. Administrative Science Quarterly, 4(2), 285-308. https://doi.org/10.2307/2392498

Karasek, R., Brisson, C., Kawakami, N., Houtman, I., Bongers, P., \& Amick, B. (1998). The Job Content Questionnaire (JCQ): An instrument for internationally comparative assessments of psychosocial job characteristics. Journal of 
Occupational Health Psychology, 3(4), 322-355. https://doi. org/10.1037/1076-8998.3.4.322

Kortum, E, Leka, S., \& Cox, T. (2011). Perceptions of psychosocial hazards, work-related stress and workplace priority risks in developing countries. Journal of Occupational Health, 53(2), 144-155. https://doi.org/10.1539/joh.O10016

Kortum, E., \& Leka, S. (2014). Tackling psychosocial risks and work-related stress in developing countries: The need for a multilevel intervention framework. International Journal of Stress Management, 21(1), 7-26. https://doi.org/10.1037/ a0035033

Kortum, E., Leka, S., \& Cox, T. (2010) Psychosocial risks and workrelated stress in developing countries: Health impact, priorities, barriers and solutions. International Journal of Occupational Medicine and Environmental Health, 23(3), 225-238. https:// doi.org/10.2478/v10001-010-0024-5

Kraemer, H. C., Stice, E., Kazdin, A., Offord, D., \& Kupfer, D. (2001). How do risk factors work together? Mediators, moderators, and independent, overlapping, and proxy risk factors. American Journal of Psychiatry, 158, 848-856. https:// doi.org/10.1176/appi.ajp.158.6.848

Lesener, T., Gusy, B., \& Wolter, C. (2019). The job demandsresources model: A meta-analytic review of longitudinal studies. Work \& Stress, 33(1), 76-103. https://doi.org/10.1080 /02678373.2018.1529065

Lorente, K., \& Yeves, J. (2016). Modelos explicativos de stress laboral. In M. J. Chambel (Ed.), Psicologia da saúde ocupacional (pp. 71-94). Factor.

Luchman, J. N. \& González-Morales, M. G. (2013). Demands, control, and support: A meta-analytic review of work characteristics interrelationships. Journal of Occupational Health Psychology, 18(1), 37-52. https://doi.org/10.1037/ a0030541

Machado, L. S., \& Macêdo, K. B. (2016). Análise bibliométrica dos estudos em clínica psicodinâmica do trabalho. Revista Subjetividades, 16(1), 9-23. https://doi. org/10.5020/23590777.16.1.9-22

Martins, S. R. (2008). Tempo antes de adoecer: relações entre saúde e os processos psicodinâmicos de reconhecimento no trabalho. In A. M. Mendes (Ed.), Trabalho e saúde: O sujeito entre emancipação e servidão (pp. 69-88). Juruá.

Merlo, A. R. C. \& Mendes, A. (2009). Perspectivas do uso da psicodinâmica do trabalho no Brasil: Teoria, pesquisa e ação. Cadernos de Psicologia Social do Trabalho, 12(2), 141-156. https://doi.org/10.11606/issn.1981-0490.v12i2p141-156

Moreno, N. P., Suárez, A. P., Cruz, Z. L., López, D. R., \& Rubiano, M. G. (2015). Bibliometric analysis of scientific output on psychosocial risk at work, 2000-2010. Diversitas: Perspectivas en Psicología, 11(1), 147-161. http://www.scielo.org.co/scielo. php?script $=$ sci_arttext\&pid $=\mathrm{S} 1794-99982015000100011$

Moreno-Jiménez, B. (2011). Factores y riesgos laborales psicosociales: Conceptualización, historia y cambios actuales. Medicina y Seguridad del Trabajo, 57, 4-19. https://doi. org/10.4321/S0465-546X2011000500002

Neffa, J. C. (2019a). Propuesta de marco teórico para estudiar los RPST (I) Primera parte: Modelos tradicionales de RPST y nuevos enfoques. Trabalho (En)Cena, 4(Esp.), 07-33. https:// doi.org/10.20873/2526-1487V4NEspecialP07

Neffa, J. C. (2019b). Propuesta de marco teórico para estudiar los RPST (II) Segunda Parte: Nuevos enfoques a partir del proceso de trabajo. Trabalho (En)Cena, 4(Esp.), 34-57. https://doi. org/10.20873/2526-1487V4NEspecialP34
O’Donnell, E., Landolt, K., Hazi, A., Dragano, N., \& Wright, B. J. (2015). An experimental study of the job demand-control model with measures of heart rate variability and salivary alpha-amylase: Evidence of increased stress responses to increased break autonomy. Psychoneuroendocrinology, 51, 24-34. https://doi.org/10.1016/j.psyneuen.2014.09.017

Organização Internacional do Trabalho (1984). Factores psicosociales en el trabajo: Naturaleza, incidencia $y$ prevención. http://www.factorespsicosociales.com/wp-content/ uploads/2019/02/FPS-OIT-OMS.pdf

Organização Internacional do Trabalho. (2010). Riesgos emergentes y nuevos modelos de prevención en un mundo de trabajo en transformación. https://www.ilo.org/wcmsp5/groups/public/--ed_protect/---protrav/---safework/documents/publication/ wcms 124341.pdf

Pacheco, T. P., \& Silva, R. M. P. D. (2018). Risco psicossocial para servidores de universidade pública na região norte do Brasil. Revista Psicologia Organizações e Trabalho, 18(1), 335-344. https://doi.org/10.17652/rpot/2018.1.13388

Portuné, R. (2012). Psychosocial risks in the workplace. Archives of Industrial Hygiene and Toxicology, 63, 123-131. https://doi. org/10.2478/10004-1254-63-2012-2212

Puente-Palacios, K. E., Porto, J. B., \& Martins, M. C. F. (2016). A emersão na articulação de níveis em Psicologia Organizacional e do Trabalho. Revista Psicologia Organizações e Trabalho, 16(4), 358-366. https://doi.org/10.17652/rpot/2016.4.12603

Rodrigues, C. M. L, \& Faiad, C. (2019). Pesquisa sobre riscos psicossociais no trabalho: Estudo bibliométrico da produção nacional de 2008 a 2017. Revista Psicologia Organizações e Trabalho, 19(1), 571-579. http://dx.doi.org/10.17652/ rpot/2019.1.15424

Ruiz, V. S., \& Araújo, A. L. L (2012). Saúde e segurança e a subjetividade no trabalho: Os riscos psicossociais. Revista Brasileira de Saúde Ocupacional, 37(125), 170-180. https:// doi.org/10.1590/S0303-76572012000100020

Sauvezon, C., Ferrieux, D., \& Priolo, D. (2019). Le stress au travail: Du paradigme des risques psychosociaux à celui de la qualité de vie au travail. Psychologie du Travail et des Organisations, 25(2), 86-99. https://doi.org/10.1016/j.pto.2018.11.002

Souza, P. C. Z., \& Athayde, M. (2006). A contribuição da abordagem clínica de Louis Le Guillant para o desenvolvimento da psicologia do trabalho. Estudos e Pesquisas em Psicologia, 6(1), 6-19. http://pepsic.bvsalud.org/pdf/epp/v6n1/v6n1a02. pdf

Stansfeld, S., \& Candy, B. (2006). Psychosocial work environment and mental health: A meta-analytic review. Scandinavian Journal of Work, Environment \& Health, 32(6), 443-462. https://doi.org/10.5271/sjweh.1050

Uribe, J. F. P. (2015). Justicia social, trabajo y factores psicosociales. In J. F. P. Uribe (Ed.), Clima y ambiente organizacional: Trabajo, salud e factores psicosociales (pp. 1-26). Manual Moderno.

Weissbrodt, R., \& Giauque, D. (2017). Labour inspections and the prevention of psychosocial risks at work: A realist synthesis. Safety Science, 100, 110-124. https://doi.org/10.1016/j. ssci.2017.02.012

Zanelli, J. C., \& Kanan, L. A. (2018). Fatores de risco, proteção psicossocial e trabalho: Organizações que emancipam ou que matam. Uniplac.

Zoni, S., \& Luchini, R. G. (2012). European approaches to work-related stress: A critical review on risk evaluation. Safety and Health at Work, 3, 43-49. https://doi.org/10.5491/ SHAW.2012.3.1.43 